\title{
The world's first clinical trial using iPS cell sheets for corneal epithelial stem cell deficiency
}

\author{
Akira Akabayashi ${ }^{1 *}$, Eisuke Nakazawa ${ }^{1}$ and Nancy S Jecker ${ }^{2,3}$ \\ ${ }^{1}$ Department of Biomedical Ethics, School of Public Health, University of Tokyo Graduate School of Medicine, Tokyo, Japan \\ ${ }^{2}$ Department of Bioethics and Humanities, University of Washington School of Medicine, Seattle, Washington, USA \\ ${ }^{3}$ University of Johannesburg, African Centre for Epistemology and Philosophy of Science, Johannesburg, South Africa
}

\begin{abstract}
Background: The world's first clinical trial using induced pluripotent stem cell sheets for corneal epithelial stem cell deficiency is underway. Elsewhere, we have analysed the protocol and argued it basically ethical.

Main points for debates: We raise three ethical points. First, the new therapy should demonstrate greater therapeutic effects when compared with other treatment methods. Second, cost is a potential barrier to widespread adoption. Third, the shortage of eye donors in Japan raises ethical concerns of fairness, as Japan relies on donors in other countries

Conclusion: While there are many issues to be debated and addressed for this new eye therapy, we believe the ophthalmology field will assume moral leadership in the pursuit of iPSC clinical research.
\end{abstract}

\section{Background}

On December 26, 2018, the review committee of Osaka University, Japan, granted conditional approval for a clinical trial to transplant corneal cell sheets from induced pluripotent stem cells (iPSC) in patients with corneal epithelial stem cell deficiency [1]. We previously objected to the protocol in a separate iPSC transplantation study for heart failure patients as ethically impermissible [2]. However, the protocol in the latest corneal trials appears to meet ethical standards very well. It appears to be fully reliable in its handling of adverse events (rejection or tumorigenicity), as transplanted corneal sheets can be easily removed. Since the new study is already underway, an ethical discussion is even more critical.

\section{Main points for debates}

Below, we raise three points for debate for this up and coming therapy.

First, the potential benefits of the study seem to warrant the risk to which research subjects are exposed. The question of whether iPSC sheet grafts can truly surpass allogeneic donated eye transplants or amniotic membranes in quality and therapeutic effects warrants rigorous scientific assessment. The subjects in this transplant study are patients with corneal epithelial stem cell deficiency. Some ophthalmology specialists note that in other cornea diseases, endothelial dysfunction is particularly salient. iPSC sheets replace mainly the epithelium. On the other hand, amniotic membrane demonstrates a record of effectiveness within certain parameters. Demonstrating greater therapeutic effects will be key to adding another future option to cornea transplant methods.
Second, the high cost of the current iPS cell sheet treatment is a potential barrier to widespread adoption. Although the research team hopes to obtain national public health insurance coverage for the procedure, current corneal graft procedures cost up to JPY 300,000 (about USD 2,700) for one eye. How much iPSC sheets can lower this cost will be key to their successful adoption.

Third, the shortage of eye donors in Japan raises ethical concerns of fairness, as Japan relies on donors in other countries. Although organ donation has been legal in Japan the supply of organ donors of all types has remained low including cornea [3]. Data from one study reported that, between 2005 and 2011 overseas donors, accounted for more than half $(53.2 \%)$ of all 1,009 corneal transplants [4]. The priority should be recruitment efforts aimed at increasing the supply of domestic donors (both cadavers and brain-dead patients).

\section{Conclusion}

Our points highlight just a few of the critical ethical issues at stake. Although the first age-related macular degeneration retina study, which was also ethically suitable, has not shown successful results yet, perhaps the ophthalmology field will assume 'moral' leadership in the pursuit of iPSC clinical research. A success story like these are

${ }^{\star}$ Correspondence to: Akira Akabayashi, Department of Biomedical Ethics, School of Public Health, University of Tokyo Graduate School of Medicine, Tokyo, Japan, E-Mail: akirasan-tky@umin.ac.jp

Key words: cornea, iPS, stem cell

Received: May 08, 2019; Accepted: May 15, 2019; Published: May 22, 2019 
exactly what the research community needs. The clinical application of iPSC transplants will be successful only once the scientific, social and ethical issues it raises are fully addressed [5].

\section{Conflict of interest}

AA is President of the Japan Association for Bioethics (JAB) and Board Member of International Association of Bioethics (IAB). This paper reflects the author's personal academic analyses and opinions and does not represent JAB's or IAB's official position.

EN and NSJ report no conflict of interest to declare.

\section{Author contributions}

AA: Conceptualization, Writing - original draft, Writing - review \& editing

EN: Conceptualization, Formal analysis, Writing - review \& editing

NJ: Conceptualization, Writing - review \& editing

\section{Funding}

The study has been funded by the Hitachi Global Foundation, the Kurata Grant, No. 1373.
The university of Tokyo research ethics committee judged that there is no need for protocol approval because this study does not use human materials.

\section{Acknowledgements}

None

\section{Data availability}

This study is an analysis of ethical arguments and does not use any data.

\section{References}

1. Osaka University research ethics committee approved iPS cornea clinical trial. (2018) Yomiuri Online. https://yomidr.yomiuri.co.jp/article/20181227-OYTET50011/

2. Akabayashi A, Nakazawa E, Jecker NS (2018) Japan must tighten up clinical trial of stem cells for heart failure. Nature 560: 431. [Crossref]

3. Akabayashi A, Nakazawa E, Ozeki R (2018) Twenty years after enactment of the Organ Transplant Law in Japan: Why are there still so few deceased donors? Transplant Proc 50: 1209-1219. [Crossref]

4. Introduction of eye bank. (2018) Department of Ophthalmology, Keio University School of Medicine. http://ophthal.med.keio.ac.jp/eyebank/kanja.html.

5. Mandai M, Watanabe A, Kurimoto Y Autologous Induced Stem-Cell-Derived Retinal Cells for Macular Degeneration. N Engl J Med 376: 1038-1046. [Crossref]

\section{Ethical approval}

